\title{
Association of SOD2, a Mitochondrial Antioxidant Enzyme, with Gray Matter Volume Shrinkage in Alcoholics
}

\author{
Vibhuti Srivastava*,', Beata Buzas ${ }^{2,3}$, Reza Momenan ${ }^{4}$, Gabor Oroszi', Attila J Pulay ${ }^{1,5}$, Mary-Anne Enoch', \\ Daniel W Hommer ${ }^{4}$ and David Goldman' \\ 'Laboratory of Neurogenetics, NIAAA, NIH, Bethesda, MD, USA; ${ }^{2}$ Mood and Anxiety Disorders Program, NIMH, NIH, Bethesda, MD, USA; \\ ${ }^{3} \mathrm{NIAAA}, \mathrm{NIH}$, Bethesda, MD, USA; ${ }^{4}$ Laboratory of Clinical and Translational Studies, NIAAA, NIH, Bethesda, MD, USA; ${ }^{5}$ Laboratory of \\ Epidemiology and Biometry, NIAAA, NIH, Bethesda, MD, USA
}

\begin{abstract}
Chronic alcoholism leads to gray matter shrinkage and induces the formation of superoxide anions $\left(\mathrm{O}_{2}^{-}\right)$that can cause neuronal cell death. The mitochondrial superoxide dismutase 2 (SOD2) enzyme is critical in the metabolism of superoxide. An Alal6Val polymorphism putatively affects SOD2 enzyme activity in vivo. Brain volumes of 76 treatment-seeking alcohol-dependent individuals were measured with a I.5T MRI. Intracranial tissue margins were manually outlined on coronal sections. Gray matter, white matter, sulcal, and ventricular CSF volumes were estimated using intensity-based K-means clustering. Alal6Val (rs4880) and a second haplotype tagging SNP, rs 10370, were genotyped. The q-value package was used to correct for multiple comparisons. In the alcoholics, cerebrospinal fluid and intra-cranial volumes showed significant differences across the six diplotype categories. The homozygous Ala I6-containing diplotype rs 10370 TT-rs4880GG was associated with lowest gray matter ratio (greater shrinkage; $p=0.005$ ). Presence of one or two copies of the low activity Ala 16 allele was a risk factor for lower gray matter volume in alcoholics below the median alcohol consumption ( $p=0.03)$ but not in alcoholics above this level. White matter ratio was associated with sex $(p=0.002)$ and lifetime total alcohol consumption $(p=0.0 \mathrm{I})$ but not with diplotypes. In this exploratory analysis, a putative functional missense variant of SOD2 appears to influence gray matter loss in alcoholics. This may be due to impaired clearance of reactive oxygen species formed as a result of alcohol exposure. The risk/protective effect was observed in alcoholics with lower levels of lifetime alcohol consumption. Highest levels of exposure may overwhelm the protective action of the SOD2 enzyme.
\end{abstract}

Neuropsychopharmacology (2010) 35, I I20-1 128; doi:I0.1038/npp.2009.2 I7; published online 30 December 2009

Keywords: alcoholism; oxidative stress; brain volume; gray matter shrinkage; SOD2; Ala I6Val

\section{INTRODUCTION}

In chronic alcoholism, structural brain alterations such as reduction in white matter, gray matter, and overall brain shrinkage have been well documented (Jernigan et al, 1991; Pfefferbaum et al, 1992; Bjork et al, 2003; Hommer, 2003; Cardenas et al, 2005; Gilman et al, 2007). These changes occur in both sexes (Jernigan et al, 1991; Bjork et al, 2003) but are more pronounced in women (Mann et al, 2005). Age-related brain degeneration is also higher in alcoholics compared with normal controls (Pfefferbaum et al, 1992, 1998). Some of these changes seem reversible, with abstinence leading to recovery of brain volume (Pfefferbaum et al, 1995; Sullivan et al, 2000; Meyerhoff et al, 2004).

*Correspondence: Dr $\vee$ Srivastava, Laboratory of Neurogenetics, National Institute on Alcohol Abuse and Alcoholism, National Institutes of Health, 5625 fishers Lane, Bethesda, MD 2085I, USA, Tel: + 130 | 443 3242, Fax: + I30 | 480 2839, E-mail: srivastavav@mail.nih.gov Received 26 June 2009; revised 29 October 2009; accepted 19 November 2009
The mechanisms of alcohol-induced brain degeneration and recovery are ill understood. Alcohol-induced degeneration is likely to be influenced by the pharmacokinetics of ethanol absorption and clearance as well as a variety of environmental factors including nutritional imbalances, particularly thiamine deficiency (Maschke et al, 2005; reviews by $\mathrm{Ke}$ and Gibson, 2004; Gibson and Zhang, 2002). Comorbid conditions such as smoking (Durazzo et al, 2007; Gazdzinski et al, 2008) and substance abuse (Bjork et al, 2003) may also be consequential. Preclinical studies have identified oxidative stress (OS) as an important origin of ethanol-induced brain damage (Crews and Nixon, 2009). Clinical studies have linked chronic alcohol abuse to enhanced oxidative damage and reduced levels of endogenous antioxidants (Lecomte et al, 1994; Peng et al, 2005). Several investigations have reported a protective effect of antioxidants against alcohol-induced brain damage (Sanchez-Moreno et al, 2003; Crews et al, 2006).

Oxidative stress-mediated damage occurs by the accumulation of free radicals (reactive oxygen (ROS) and nitrogen (RNS) species), which are the by-products of 
cellular oxidation and may have an important role in cell regulatory and signaling processes at moderate concentrations (Droge, 2002). The damaging effects of free radicals have been linked to a wide-range of pathological conditions, including diabetes (Lenzen, 2008), atherosclerosis (Finsterer, 2007), macular degeneration (Beatty et al, 2000), and certain forms of cancer (Toyokuni, 2008). Also, OS has been implicated in neurodegenerative disorders such as Alzheimer's disease (Moreira et al, 2008) and Parkinson's disease (Chinta and Andersen, 2008), and neuropsychiatric disorders such as schizophrenia (Yao et al, 2001).

Excessive environmental oxidative challenges may result in the increased production and insufficient clearance of free radicals that in turn can cause cell degeneration in many tissues. Heavy alcohol consumption can represent such a challenge because ethanol enhances ROS production in brain (Sun and Sun, 2001) and other tissues, leading to an imbalance in anti- and pro-oxidant levels. Of all the ROS, the superoxide anion radical is one of the most important free radicals. It is neutralized by the superoxide dismutases (SODs), a family of enzymes that convert this unstable anion to the relatively more stable and less toxic hydrogen peroxide. Superoxide dismutase 2 (SOD2; manganese superoxide dismutase) is a mitochondrial antioxidant enzyme that is induced and regulated by ROS through a signaling cascade (Weisiger and Fridovich, 1973; Storz et al, 2005). Neuronal cell death has been reported in Sod2-null mice (Hinerfeld et al, 2004), and drosophila Sod2 mutants exhibit increased apoptosis in the central nervous system (CNS) and early-onset neurodegeneration (Paul et al, 2007). Evidence shows that the SOD2 enzyme may also be pivotal in alcohol-induced, OS-related structural brain loss. SOD activity in brain and liver is reduced in chronic alcoholic patients (Huang et al, 2008) and alcoholics in withdrawal (Tsai et al, 1998).

These reductions in SOD activity seen in alcoholics might be accounted for by decreased levels of gene expression and/or alterations in protein structure that are either induced or inherited. A common SOD2 missense variant (Ala16Val, rs4880) has been identified that putatively reduces the activity of the SOD2 enzyme (ShimodaMatsubayashi et al, 1996, 1997; Bastaki et al, 2006; Martin et al, 2009). We hypothesized that this SOD2 polymorphism might predict vulnerability or resilience to reductions in brain volume consequent to ethanol exposure in chronic alcoholics.

\section{MATERIALS AND METHODS}

\section{Participants}

Seventy-six treatment-seeking alcohol-dependent individuals (61 men, 15 women; mean (SD) age $=37.9$ (7.9) years) underwent 1.5T MRI scans and were genotyped for SOD2. These participants were in-patients at the National Institute on Alcohol Abuse and Alcoholism (NIAAA) inpatient unit at the NIH Clinical Center, Bethesda, MD. The following were the inclusion criteria: IQ $>80$ and a diagnosis of alcohol dependence (and not alcohol abuse). Exclusion criteria were as follows: a history of delirium tremens, gross neurological disorders, dementia, Korsakoff's disease, thiamine deficiency, head injury and comorbid psychiatric disorders (including substance abuse) other than nicotine dependence. The sample was predominantly European Caucasian $(n=51 ; 67 \%)$ and also included 23 African Americans (30\%), and 2 Hispanic (3\%) individuals. Information on recent and chronic alcohol use was obtained from structured research questionnaires (Eckardt et al, 1978). Lifetime alcohol consumption was estimated in kilograms, summing all alcohol consumption including the periods where ingestion did not reach 90 drinks per month. Mean lifetime alcohol consumption was $614.4 \mathrm{~kg}$ $(\mathrm{SD}=516.7)$ and the median was $420.6 \mathrm{~kg}$. The majority of alcoholics were current smokers; there were seven nonsmokers. The mean years of smoking was 20.5 years $(\mathrm{SD}=11.2)$. On the basis of a physical examination, blood chemistry, negative urine screen, and general medical history, all the alcoholics were found to be medically healthy at the time of admission.

In our larger sample set, $44 \%$ of the patients showed mildto-moderate elevation in liver transaminase levels and only 9\% showed mildly elevated levels after 3 weeks of admission. None of the patients showed any sign of common liver disease such as hepatitis or cirrhosis (Hommer et al, 2001). The levels of liver enzymes measured within first $24 \mathrm{~h}$ of admission, namely, aspartate aminotransferase, alanine transaminase, gamma-glutamyl transpeptidase, and the mean corpuscular volumes, were available for the patients included in this study.

This data set of alcoholic patients that are the focus of this study was drawn from a larger sample that has previously been shown to have significant volume reductions as a result of alcohol exposure (Bjork et al, 2003; Hommer, 2003; Gilman et al, 2007).

We have verified that this genetic subsample, which was selected only on the basis of DNA availability, shows similar volume reductions as that larger sample of alcoholic patients. A detailed description of the study sample has been provided elsewhere (Bjork et al, 2003; Hommer, 2003; Gilman et al, 2007).

To verify the effect of the alcohol exposure in this data set on brain volume loss, we also included 69 healthy agematched controls (37 men, 32 women; mean (SD) age $=36.16 \quad(9.29)$ years $)$ with no history of alcohol dependence or abuse or other psychiatric disorders. The controls included 54 European Caucasians (78\%), 13 African Americans (19\%), and 2 Hispanic (3\%). Overall, 45 were non-smokers, 8 were smokers, while no data on smoking were available for 16 control individuals. The mean (SD) lifetime alcohol consumption was 10.85 (12.73) $\mathrm{kg}$ and mean (SD) years of smoking for the controls was1.19 (3.98) years. DNA was unavailable for controls.

Participants were assessed with the Structured Clinical Interview for either DSM-III-R or DSM-IV. The protocol and consent forms were approved by the NIAAA IRB, and all participants provided informed consent. A detailed description of the sample from which this data set was drawn is provided elsewhere (Gilman et al, 2007; Schottenbauer et al, 2007).

\section{MRI and Brain Volume Calculations}

These alcoholic in-patients were scanned not earlier than 3 weeks and not later than 5 weeks after last alcohol use. 
Brain scanning was performed with a 1.5T MRI (GE Medical Systems, Milwaukee, Wisconsin) using a fast spoiledGRASS (FSPGR) sequence. High contrast $2 \mathrm{~mm}$ thick T1-weighted coronal images were obtained and tissue margins were defined manually on coronal sections. Cerebrum and cerebrospinal fluid (CSF) spaces were included in intra-cranial volume (ICV). Brain tissue was automatically segmented (Momenan et al, 1997) into gray matter, white matter, sulcal CSF, and ventricular CSF. All the images were acquired with a $256 \times 256$ matrix and a $240 \mathrm{~mm}$ field of view. Each volumetric brain consisted of 124 coronal slices with voxel size of $0.9375 \times 0.9375 \times$ $2.0 \mathrm{~mm}^{3}$. All brain volume measures are in milliliters. Total cerebral brain volume was calculated as the sum of gray and white matter volumes. Total brain ratio was defined as the ratio of total cerebral brain volume to ICV; gray matter ratio as the ratio of gray matter volume to ICV; and white matter ratio as the ratio of white matter volume to ICV. These ratios are reflective of respective tissue shrinkage where a lower ratio (marginal mean) indicates higher shrinkage and vice versa.

Further details about brain volume measurement are provided elsewhere (Momenan et al, 1997; Gilman et al, 2007; Schottenbauer et al, 2007).

\section{Genetic Analyses}

Genomic DNA was isolated from blood using standard protocols. Two SOD2 SNPs, namely, rs10370G/T ( $3^{\prime}$ near gene) and rs4880G/A (exon 2; Ala16Val), were selected because Ala16Val is the putative functional variant and together these two SNPs capture (tag) the maximum haplotypic diversity within the $27.9 \mathrm{~Kb}$ region that includes the SOD2 coding sequence and its flanking regions in European Caucasians (data not shown). These two tags were not significantly correlated with each other in either of the populations; $r^{2}$ between rs4880 and rs10370 was 0.26 among European-American population and 0.13 among AfricanAmerican sample set. Genotyping was performed using $5^{\prime}$ exonuclease assays: Taqman Assays-on-Demand (Applied Biosystems, Foster City, California): C_1362062_10 for rs10370 and C_8709053_10 for rs4880. Genotyping was performed according to the manufacturer's protocol and genotypes were determined at the end point using an ABI 7900HT Sequence Detection System. Genotyping accuracy was determined empirically by duplicate genotyping of $10 \%$ of the samples selected randomly. The error rate was $<0.005$. Genotypes conformed to Hardy-Weinberg equilibrium.

\section{Statistical Analyses}

Statistical tests were performed using the JMP-SAS package (SAS institute; Cary, NC). Correlations between the brain measures were assessed using Pearson's bivariate correlation test. Assumptions of ANOVA were assessed by Shapiro-Wilk $W$-test for normality of distribution and Welch ANOVA testing for homogeneity of variance. If either assumption was violated, a less powerful, nonparametric Wilcoxon rank sums test (Kruskal-Wallis $H$-test) was used. The standard least square method was used to construct the linear regression models with respective brain volume measures as the dependent variables and diplotypes, sex, ethnicity, age, lifetime total alcohol consumption, and total lifetime smoking years as covariates. All the variables were simultaneously entered in the model. Tukey's HSD analysis was used as a posterior test. To estimate the error rate stemming from multiple testing, we assessed the false discovery rate (FDR) using Benjamini and Hochberg (B\&H) method (1995). Briefly, the $q$-value is defined as the smallest modified $p$-value among tests with equal or larger rank for each test considered. $\mathrm{B} \& \mathrm{H}$ method is considered to be independent of the $p$-value distribution pattern because the $q$-values have the same ordering as the $p$-values (implemented in $\mathrm{R}$-package version 1.1.1, 2008). This approach provides conservative estimates of the FDR.

Haplotypes were constructed and diplotypes were inferred using PHASE version 2.0.2 (Stephens et al, 2001; Stephens and Donnelly, 2003).

\section{RESULTS}

\section{Brain Volume Changes in Alcoholics Compared with Controls}

The mean (SD) brain volume measures for cases and controls are presented in Table 1. From Figure 1 it can be seen that the gray and white matter ratios showed stronger negative correlations with age and alcohol exposure among alcoholics compared with the controls. As previously stated, a lower brain ratio indicates higher shrinkage and vice versa. As expected, age was negatively correlated with gray matter ratio $\left(r_{\text {alcoholics }}=-0.60 ; r_{\text {controls }}=-0.42\right)$ and white matter ratio $\left(r_{\text {alcoholics }}=-0.14 ; r_{\text {controls }}=-0.08\right)$. Lifetime alcohol exposure also showed the same trend of higher negative correlations in alcoholics compared with controls with both gray matter ratio $\left(r_{\text {alcoholics }}=-0.47\right.$; $\left.r_{\text {controls }}=-0.25\right)$ and white matter ratio $\left(r_{\text {alcoholics }}=-0.25\right.$; $\left.r_{\text {controls }}=-0.14\right)$. All these correlations achieved nominal significance.

Table I Brain Volume Measurements ${ }^{\mathrm{a}}(\mathrm{ml})$ for Alcoholics and Controls

\begin{tabular}{|c|c|c|}
\hline Brain volume measure & $\begin{array}{l}\text { Controls } \\
(N=69)\end{array}$ & $\begin{array}{l}\text { Alcoholics } \\
(N=76)\end{array}$ \\
\hline & \multicolumn{2}{|c|}{ Mean (SD) } \\
\hline Gray matter volume & $581.5(56.6)$ & $573.2(54.1)$ \\
\hline White matter volume & $513.0(66.0)$ & $502.9(53.3)$ \\
\hline $\begin{array}{l}\text { Total brain volume (gray matter } \\
\text { volume+white matter volume) }\end{array}$ & \multicolumn{2}{|c|}{1094.5 (| | 15.7) I076.| (I05.|) } \\
\hline CSF volume & $220.3(47.9)$ & $230.6(49.0)$ \\
\hline Ventricle volume & $17.0(10.3)$ & |9.1 (9.8) \\
\hline $\begin{array}{l}\text { Intra cranial volume (ICV) (total brain } \\
\text { vol+CSF+ventricular vol) }\end{array}$ & \multicolumn{2}{|c|}{1331.8 (| 53.7)|325.8 (|24.6) } \\
\hline White matter ratio (white matter volume/ICV) & $0.39(0.02)$ & $0.38(0.02)$ \\
\hline Gray matter ratio (gray matter volume/lCV) & $0.44(0.02)$ & $0.43(0.02)$ \\
\hline Total brain ratio (total brain volume/lCV) & $0.82(0.03)$ & $0.81(0.03)$ \\
\hline
\end{tabular}

aMean (SD). 


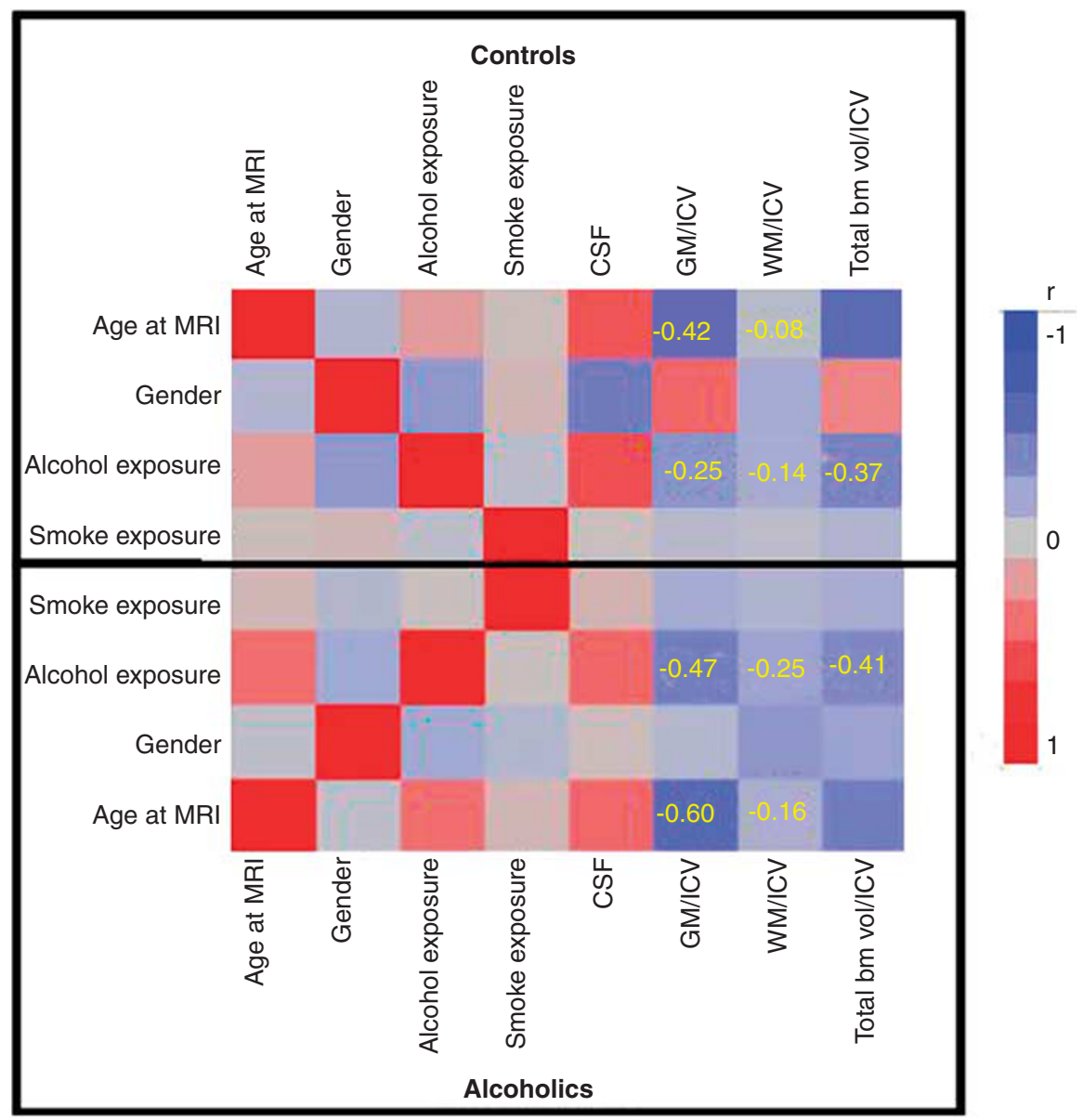

Figure I Correlation analysis (Numerical values in the squares represent the magnitude of correlation coefficient; alcohol exposure refers to the lifetime total alcohol consumption (kg); smoke exposure refers to the total lifetime smoking years.) of age and alcohol consumption with brain ratios in alcoholics and controls.

\section{SOD2 Associations with Brain Volume Measures in Alcoholics}

Allele frequencies for both SNPs were compared with appropriate NCBI reference populations (http://www. ncbi.nlm.nih.gov/SNP) (European Caucasian Americans to Europeans and African Americans to Africans) and no significant differences were observed $(p=0.11$ and 0.72$)$ (Table 2).

\section{SOD2 Diplotype Associations with Brain Volume Measures}

Three common SOD2 haplotypes and six diplotypes were observed (Table 2). The distributions of brain volume measures were assessed for all diplotypes separately. CSF and IC volumes showed nominally significant differences across the diplotypes (Table 3 ). The lowest mean CSF and IC volumes were observed for the TT-AA(Val/Val) diplotype compared with the other five diplotypes (Table 3 ). However, the other two Val/Val-containing diplotypes (TG-AA(Val/Val) and GG-AA(Val/Val) had higher CSF and IC volumes suggesting that the diplotype effect on brain volume may be dependent on both SNPs and not just the missense variant.
Only two brain volume measures, namely, gray and white matter ratios, followed ANOVA assumptions enabling linear regression models to be constructed to analyze the influence of SOD2 diplotype variation. Potentially confounding factors (sex, ethnicity, age, lifetime total alcohol consumption, and total lifetime smoking years) were included as independent variables in the model. We found that white matter ratio was lower in women than men (a lower brain ratio indicates higher shrinkage): mean ratio $=0.37 v s 0.38$, respectively, $p=0.002$; and in individuals with higher lifetime total alcohol consumption $(p=0.01 ; r=-0.23)$. SOD2 diplotypes did not predict white matter ratio.

Gray matter ratio was significantly associated with age $(p<0.0001 ; r=-0.62$ and lifetime total alcohol consumption $(p=0.003 ; r=-0.45$, ie, increased age and higher consumption was correlated with greater shrinkage). The TT-GG(Ala/Ala) diplotype was also significantly associated with gray matter ratio $(p=0.005)$ (Table 4$)$. The amount of variance accounted by the associated variables was $19 \%$ for age, $9 \%$ for diplotypes, and $8 \%$ for lifetime alcohol consumption. Table 3 shows that, compared with the other five diplotypes, alcoholics with the TT-GG(Ala/Ala) diplotype had the lowest mean gray matter ratio (0.42) (and therefore the highest gray matter shrinkage), whereas alcoholics with the TT-AA(Val/Val) and TG-AA(Val/Val) 
Table 2 SOD2 Haplotype, Diplotype, and Allele Frequencies in the 76 Alcoholics

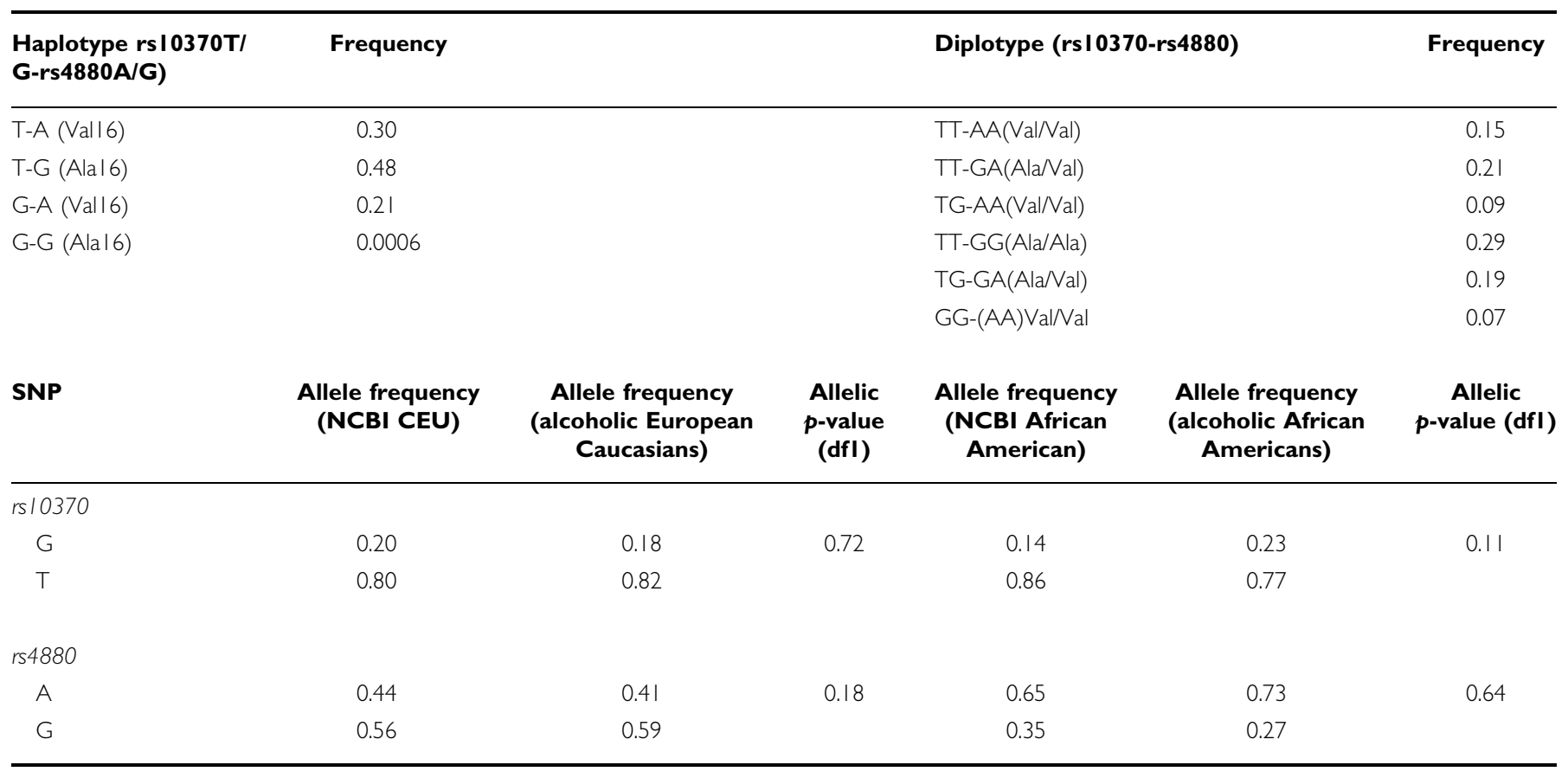

diplotypes had the highest gray matter ratio (0.44). Supplementary Figure 1 shows the distribution of Ala16 Val genotypes versus gray matter ratio in alcoholics. Coronal brain sections representing segmented gray matter in two alcoholics, one with the TT-GG(Ala/Ala) diplotype and one with the TT-AA(Val/Val) diplotype are shown in Figure 2.

\section{Dosage Effect of Ala16Val Alleles on Gray Matter Ratio}

Following the observation of highest gray matter shrinkage among alcoholics who were homozygous for the Ala16containing diplotype [TT-GG(Ala/Ala)], patients were coded as having 0,1 , or 2 copies of the Ala16 allele and were grouped on the basis of median lifetime alcohol consumption. This was an exploratory analysis, the aim of which was to determine whether an interaction between genetic variation and higher and lower alcohol consumption would have an effect on gray matter shrinkage. We chose a median split as the distribution of lifetime alcohol consumption was skewed. Absence of the Ala16 allele was protective as it was associated with higher gray matter ratio (lower shrinkage) in the lower drinking category $(p=0.03)$ but not in the higher drinking category (Table 5). A double dose of the Ala16 allele appeared to be a nominal risk factor for highest gray matter shrinkage in low drinking category (lowest mean for gray matter/ICV ratio $=0.43$ in this category). Figure 3 depicts the relative gray matter shrinkage (1-ratio of gray matter volume/ICV) across allele dosage and as a function of lifetime alcohol consumption.

\section{Population Stratification}

The allele frequencies of rs4880 differ between the two major ethnic groups in our sample and this may lead to stratification (Table 2). Therefore, we performed a stratified analysis to rule this out. When similar regression models were constructed for Europeans and African Americans separately, the same diplotype was observed to be associated (Supplementary Table 2). Following this observation, we used ethnicity as a covariate in all the regression models in this study to account for differential allele frequencies in the sample set.

\section{Single Marker Analyses}

Similar linear regression models were constructed taking into account the individual SNPs rather than the diplotypes, and age, ethnicity, sex, lifetime total alcohol consumption, and total years of smoking were included as independent variables in the model. rs4880 G/A ( $p=0.002$; $p$ for $r s 4880$ $\mathrm{AA}[\mathrm{Val16} / \mathrm{Val16}]=0.02)$, age at MRI $(p<0.0001)$, and lifetime total alcohol consumption $(p=0.003)$ were significant predictors of gray matter ratio $(r=0.78)$. Although rs 10370 by itself was not associated with gray matter ratio, however as mentioned earlier, only two diplotypes, namely, TT-AA (Val/Val) and TG-AA (Val/Val) showed highest gray matter ratio $(0.44$; Table 3$)$. This indicated that both the SNPs together may be important for gray matter shrinkage. As both these SNPs act as tags for the SOD2 locus, a diplotype-based analysis was expected to be more efficient in capturing the maximum genetic variation at this locus.

\section{Liver Function as a Possible Mediator of Oxidative Stress}

To assess the possibility of liver function as a modifying variable for gray matter shrinkage, we included the levels of aspartate aminotransferase, alanine transaminase, gammaglutamyl transpeptidase, and the mean corpuscular volumes as covariates in the original regression model in addition to diplotypes, sex, ethnicity, age, lifetime total alcohol 
Table 3 Brain Volume Measures ${ }^{\mathrm{a}}$ Among the Alcoholics Across Diplotype Categories

\begin{tabular}{|c|c|c|c|c|c|c|c|}
\hline Diplotypes & $\begin{array}{l}\text { TT-AA } \\
\text { (Val/Val) }\end{array}$ & $\begin{array}{l}\text { TT-GA } \\
\text { (Ala/Val) }\end{array}$ & $\begin{array}{l}\text { TT-GG } \\
\text { (Ala/Ala) }\end{array}$ & $\begin{array}{l}\text { TG-AA } \\
\text { (Val/Val) }\end{array}$ & $\begin{array}{l}\text { TG-GA } \\
\text { (Ala/Val) }\end{array}$ & $\begin{array}{l}\text { GG-AA } \\
\text { (Val/Val) }\end{array}$ & Wilcoxon rank \\
\hline$N$ & 12 & 16 & 22 & 7 & 15 & 4 & sums test $p$ \\
\hline & \multicolumn{7}{|c|}{ Mean (SD) } \\
\hline Gray matter volume ${ }^{b}$ & $539.9(35.8)$ & $575.4(42.8)$ & $577.9(50.6)$ & $586.9(76.7)$ & $574.9(69.5)$ & $607.9(22.4)$ & $\chi^{2}=7.79 ; p=0.17$ \\
\hline White matter volume ${ }^{b}$ & $471.7(30.7)$ & $507.3(44.8)$ & $507.2(46.7)$ & $510.3(70.8)$ & $504.8(74.4)$ & $534.7(29.0)$ & $\chi^{2}=7.36 ; p=0.19$ \\
\hline CSF volume ${ }^{b}$ & $197.6(34.9)$ & $223.8(44.7)$ & $259.8(45.8)$ & $215.4(59.3)$ & $226.7(46.1)$ & $237.9(47.8)$ & $\chi^{2}=13.37 ; p=0.02$ \\
\hline Ventricle volume ${ }^{b}$ & | $3.8(4.2)$ & $19.2(13.7)$ & $20.6(7.7)$ & $16.5(7.8)$ & $20.8(11.8)$ & $24.4(6.4)$ & $\chi^{2}=9.30 ; p=0.10$ \\
\hline $\begin{array}{l}\text { Intra cranial volume }(\mathrm{ICV})^{\mathrm{b}} \\
\text { (total brain vol+CSF+ventricular vol) }\end{array}$ & $1223.2(69.6)$ & | $325.7(\mid 09.1)$ & | 365.5 (|09.8) & | $329.2(153.7)$ & I $327.2($ ( 54.6$)$ & | $404.9(74.1)$ & $\chi^{2}=13.80 ; p=0.02$ \\
\hline $\begin{array}{l}\text { Total brain ratio }{ }^{\text {(total }} \\
\text { brain volume/ICV) }\end{array}$ & $0.83(0.03)$ & $0.82(0.03)$ & $0.79(0.03)$ & $0.83(0.05)$ & $0.81(0.03)$ & $0.81(0.03)$ & $\chi^{2}=10.23 ; p=0.07$ \\
\hline
\end{tabular}

a Mean (SD).

biolated the assumption of homogeneity of variance (Welch ANOVA) and/or normal distribution (Shaipro-Wilk test; data not shown).

'Did not violate the assumptions of normal distribution and homogeneity of variance in alcoholics, so used in regression models; see Table 5 .

consumption, and total lifetime smoking years as independent variables (with gray matter ratio as the dependent variable). None of the included liver enzymes and mean corpuscular volumes were associated with shrinkage $(p>0.05)$, indicating that the gray matter shrinkage may not be mediated by the liver damage in our sample set.

\section{Corrections for Multiple Comparisons}

All the $p$-values and associated $q$-values are presented in Supplementary Table 1 . The B\&H FDR $q$-values for the 39 tests ranged from 0.002 to 0.99 . Seven $p$-values including the one emerging from the association of diplotype with gray matter shrinkage had a $q$-value less than 0.07 .

\section{DISCUSSION}

In the study sample, both advanced age and high alcohol consumption were correlated with higher gray and white matter shrinkage, and this is consistent to previous reports (Pfefferbaum et al, 1998; Gilman et al, 2007). The main finding of this study was a significant effect of SOD2 genetic variation on brain volume changes among chronic alcoholics.

The putative functional Ala16Val (rs4880G/A) SNP was individually associated with gray matter ratio. However, we also included another haplotype-tagging SNP rs10370 and constructed diplotypes, because these two tagging SNPs together capture the common haplotypes that represent the SOD2 gene region. This analysis has the advantage that it may detect effects of an unknown functional locus in the region.

There is evidence that alcohol exposure leads to inadequate ROS clearance resulting in toxicity and organ damage with special relevance in the CNS (Huang et al, 2008). Therefore, genetic variants impairing ROS clearance could be risk factors in alcohol-associated brain damage. On balance, and despite some not completely reconciled data, the SOD2 Ala16Val missense substitution seems to represent such a candidate locus. The Val16 allele was predicted to be inefficiently transported (ShimodaMatsubayashi et al, 1996, 1997) and this was seemingly confirmed experimentally when the Val16 and Ala16 SOD2 leader sequences were fused with a mouse DHFR reporter protein (Hiroi et al, 1999). However, in vivo studies using human tissues have yielded a different picture; homozygosity for Ala16 resulted in 39\% lower SOD2 activity in erythrocytes of 231 young non-smoking adults (Bastaki et al, 2006). These findings were recently replicated in human hepatocytes (Martin et al, 2009). Our results showing greater gray matter volume reductions in Ala16/ Ala16 homozygotes are consistent with the in vivo functional data that Ala16 is a lower activity allele. Speculatively, the reduced efficiency of the Ala16 allele could lead to a larger ROS load in the brains of chronic alcoholics. Even if Ala16 is more efficiently transported it may nevertheless be less active.

The association of SOD2 with gray matter volume was driven by the mean difference in gray matter ratio between diplotypes containing different numbers of Ala16 alleles, with the Ala16/Ala16 homozygotes lowest in gray matter ratio and three diplotypes missing the Ala16 allele including two with highest gray matter ratios. However, the Ala16 haplotype was only a risk factor among alcoholics with alcohol consumption below the median. In fact, these alcoholics have suffered high lifetime exposures, with a mean lifetime exposure of $614.4 \mathrm{~kg}$. Another way of looking at the data is that the seemingly fully functional Val16 SOD2 enzyme can have a protective role against ROS in chronic alcoholism, but this may be overwhelmed at higher levels of exposure. Nevertheless, in the overall model incorporating 
Table 4 Factors Affecting Reduction in Gray and White Matter Volumes (Shrinkage) Among Chronic Alcoholics

\begin{tabular}{|c|c|c|c|c|c|c|c|c|c|c|c|c|c|}
\hline \multirow[t]{2}{*}{ Dependent variable } & \multirow{2}{*}{$\begin{array}{c}R^{\mathbf{a}} \\
\text { Total }_{\text {model }^{\mathbf{b}}}\end{array}$} & \multicolumn{2}{|c|}{$\begin{array}{c}\text { Diplotype } \\
\text { TT-AA(Val/Val) }\end{array}$} & \multicolumn{2}{|c|}{$\begin{array}{c}\text { Diplotype } \\
\text { TT-GG(Ala/Ala) }\end{array}$} & \multicolumn{2}{|c|}{ Sex } & \multicolumn{2}{|c|}{ Age } & \multicolumn{2}{|c|}{$\begin{array}{l}\text { Lifetime total } \\
\text { alcohol consumption }\end{array}$} & \multicolumn{2}{|c|}{$\begin{array}{l}\text { Total lifetime } \\
\text { smoking years }\end{array}$} \\
\hline & & t-ratio & $p$ & t-ratio & $p$ & t-ratio & $p$ & t-ratio & $p$ & t-ratio & $p$ & t-ratio & $p$ \\
\hline $\begin{array}{l}\text { White matter shrinkage } \\
\text { (white matter volume/lCV) }\end{array}$ & 0.64 & 1.07 & 0.28 & -1.65 & 0.11 & -3.28 & 0.002 & -0.49 & 0.62 & -2.61 & 0.01 & -1.49 & 0.14 \\
\hline
\end{tabular}

${ }^{a}$ The value of $R$ equals the amount of variance explained by all independent variables (diplotype, sex, ethnicity, age, lifetime total alcohol consumption, and total lifetime smoking years) included in the model.

bhole model and key regression variable plots are shown in Supplementary Figure 2.

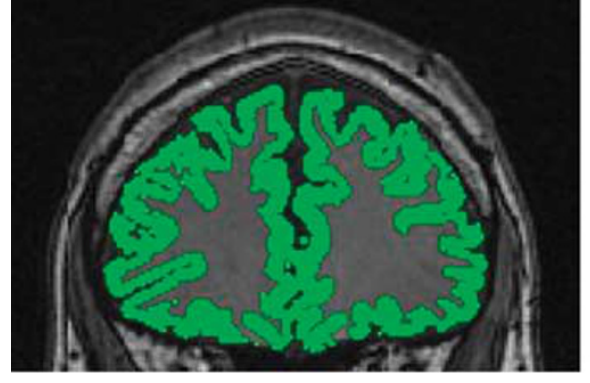

Diplotype rs10370TT-rs4880GG (Ala16Ala)

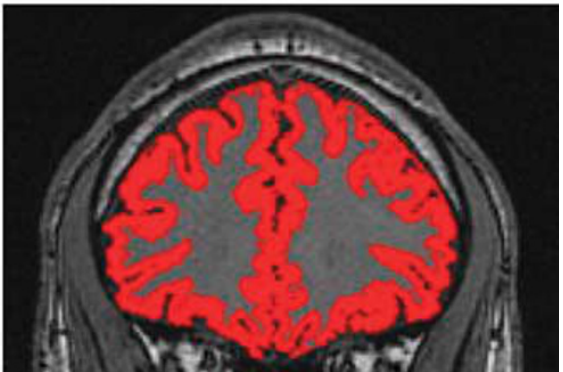

Diplotype rs10370TT-rs4880AA (Val16Val)

Figure 2 Coronal brain sections representing segmented gray matter (Segmented gray matter in green is from the brain of alcoholic having risk diplotype rs $10370 T$-rs4880GG that was associated with lower gray matter ratio (and higher gray matter shrinkage) in the sample set; gray matter segmentation in risk diplotype (green) reflects greater CSF space compared with protective diplotype (red) in case of these two particular alcoholics.) in two chronic alcoholics containing predisposing and protective SOD2 diplotypes.

Table 5 Effect of Ala 6 Allele Copy Number on Gray Matter Ratio in Low and High Drinking Categories

\begin{tabular}{|c|c|c|c|c|c|c|c|c|c|c|}
\hline \multirow{3}{*}{$\begin{array}{l}\text { Dependent variable } \\
\text { Gray matter ratio } \\
\text { (gray matter volume/ICV) }\end{array}$} & \multicolumn{5}{|c|}{ Less than median drinking category ${ }^{a}$} & \multicolumn{5}{|c|}{ More than median drinking category } \\
\hline & \multirow{2}{*}{$\begin{array}{c}\text { Total } \\
\text { model } \\
R^{\mathbf{b}}\end{array}$} & \multicolumn{2}{|c|}{ Ala 16 (0 copies) } & \multicolumn{2}{|c|}{ Ala I6 (I copy) } & \multirow{2}{*}{$\begin{array}{c}\text { Total } \\
\text { model } \\
R^{\mathrm{b}}\end{array}$} & \multicolumn{2}{|c|}{ Ala I 6 (0 copies) } & \multicolumn{2}{|c|}{ Ala I6 (I copy) } \\
\hline & & t-ratio & $P$ & t-ratio & $p$ & & t-ratio & $p$ & t-ratio & $p$ \\
\hline & 0.77 & 2.23 & $0.03^{c}$ & 0.41 & 0.68 & 0.59 & $|.5|$ & 0.14 & 0.83 & 0.41 \\
\hline
\end{tabular}

${ }^{a}$ Age was also a significant predictor of gray volume ratio in both the categories.

${ }^{b}$ The value of $R$ equals the amount of variance explained by all independent variables (Ala 16 dose, sex, age, and total lifetime smoking years) included in the model. 'Patients with zero copies showed significantly higher marginal mean (0.45) compared with those with two copies $(0.43)$.

sex, age, ethnicity, lifetime total alcohol consumption, and total lifetime smoking years (Table 4), SOD2 diplotype was a significant predictor of gray matter ratio (shrinkage) among alcoholics, with patients homozygous for Ala16 having a lower gray matter ratio.

A notable observation is that the diplotypes predicted gray matter ratio but not the white matter ratio. Alcoholism has been shown to result in altered cognition (review by Uekermann and Daum, 2008) and white matter loss has also been linked to cognitive abilities (Fields, 2008). Whether our results have any implication for neurocognition is difficult to predict. But one possible explanation is that all brain regions/cell types may not be equally susceptible to alcohol-induced damage. One study showed regional differences in SOD2 distribution in human brain (Zhang et al, 1994) and thus consequentially different ROS loads.

This exploratory analysis has a few limitations. There is heterogeneity of age and gender effect between the study populations, as evident from Supplementary Table 2. But it is difficult to ascertain if this difference arises due to a 'potential confound' or simply due to the small sample sizes. Especially in the context of brain imaging studies, it is difficult to achieve the desired/appropriate effects across all the study groups due to constraints of sample sizes. As mentioned earlier, another limitation of this study was unavailability of DNA and SOD2 genotypes from controls. 
Grouping variable: Ala16 copy number

Split by: Median lifetime alcohol consumption

Error bars: \pm 1 Standard Error (s)

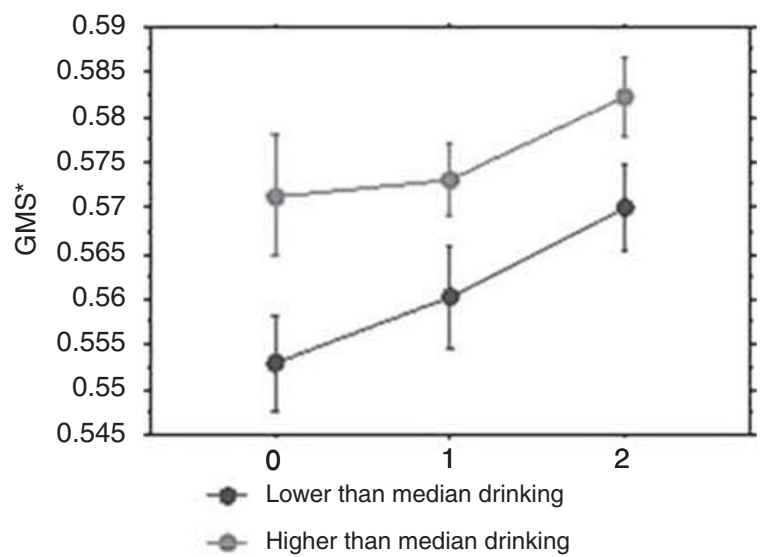

Figure 3 Relative GMS (Does not reflect absolute GMS (gray matter shrinkage); values calculated as I - (ratio of gray matter volume to ICV) for easy readability. $X$ axis represents the Alal 6 copy number.) across the Alal 6 dosage as a function of lower and higher than median lifetime alcohol consumption.

Also, because this was a retrospective study in chronic alcoholics, we could not correlate our results with SOD2 levels/activity. But we observed significant association of SOD2 diplotypes, which was adjusted for possible confounding factors, such as age, sex, and ethnicity. Following the adjustment for multiple comparisons, our results suggest that by declaring all the tests with $p$-value less than $\alpha=0.05$ as significant, the expected rate of false discoveries will still be less than $7 \%$. Given such a low FDR, absence of a mediating effect of liver damage on gray matter shrinkage, and consistence with the function of SOD2 enzyme and the probable effect of the Ala16 allele, our results merit replication.

In conclusion, Ala16Val, a putative functional genetic variation in the SOD2 gene, may influence gray matter loss in chronic alcoholics, an effect modulated by the level of alcohol consumption.

\section{ACKNOWLEDGEMENTS}

We thank Ms Pei-Hong Shen for advice on corrections for multiple comparisons and Michael Kerich for providing technical assistance.

\section{DISCLOSURE}

This work was supported by the Intramural Research Program of the National Institute on Alcohol Abuse and Alcoholism, NIH. The author(s) declare that no financial support or compensation has been received from any individual or corporate entity over the past 3 years for research or professional service and there are no personal financial holdings that could be perceived as constituting a potential conflict of interest.

\section{REFERENCES}

Bastaki M, Huen K, Manzanillo P, Chande N, Chen C, Balmes JR et al (2006). Genotype-activity relationship for Mn-superoxide dismutase, glutathione peroxidase 1 and catalase in humans. Pharmacogenet Genomics 16: 279-286.

Beatty S, Koh H, Phil M, Henson D, Boulton M (2000). The role of oxidative stress in the pathogenesis of age-related macular degeneration. Surv Ophthalmol 45: 115-134.

Benjamini Y, Hochberg Y (1995). Controlling the false discovery rate: a practical and powerful approach to multiple testing. J Royal Stat Soc Series B 57: 289-300.

Bjork JM, Grant SJ, Hommer DW (2003). Cross-sectional volumetric analysis of brain atrophy in alcohol dependence: effects of drinking history and comorbid substance use disorder. Am J Psychiatry 160: 2038-2045.

Cardenas VA, Studholme C, Meyerhoff DJ, Song E, Weiner MW (2005). Chronic active heavy drinking and family history of problem drinking modulate regional brain tissue volumes. Psychiatry Res 138: 115-130.

Chinta SJ, Andersen JK (2008). Redox imbalance in Parkinson's disease. Biochem Biophys Acta 1780: 1362-1367.

Crews F, Nixon K, Kim D, Joseph J, Shukitt-Hale B, Qin L et al (2006). BHT blocks NF-kappaB activation and ethanol-induced brain damage. Alcohol Clin Exp Res 30: 1938-1949.

Crews FT, Nixon K (2009). Mechanisms of neurodegeneration and regeneration in alcoholism. Alcohol Alcohol 44: 115-127.

Droge W (2002). Free radicals in the physiological control of cell function. Physiol Rev 82: 47-95.

Durazzo TC, Gazdzinski S, Meyerhoff DJ (2007). The neurobiological and neurocognitive consequences of chronic cigarette smoking in alcohol use disorders. Alcohol Alcohol 42: 174-185.

Eckardt MJ, Parker ES, Noble EP, Feldman DJ, Gottschalk LA (1978). Relationship between neuropsychological performance and alcohol consumption in alcoholics. Biol Psychiatry 13: 551-565.

Fields RD (2008). White matter in learning, cognition and psychiatric disorders. Trends Neurosci 31: 361-370.

Finsterer J (2007). Is atherosclerosis a mitochondrial disorder? Vasa 36: 229-240.

Gazdzinski S, Durazzo TC, Yeh PH, Hardin D, Banys P, Meyerhoff DJ (2008). Chronic cigarette smoking modulates injury and short-term recovery of the medial temporal lobe in alcoholics. Psychiatry Res 162: 133-145.

Gibson GE, Zhang H (2002). Interactions of oxidative stress with thiamine homeostasis promote neurodegeneration. Neurochem Int 40: 493-504.

Gilman JM, Bjork JM, Hommer DW (2007). Parental alcohol use and brain volumes in early- and late-onset alcoholics. Biol Psychiatry 62: 607-615.

Hinerfeld D, Traini MD, Weinberger RP, Cochran B, Doctrow SR, Harry J et al (2004). Endogenous mitochondrial oxidative stress: neurodegeneration, proteomic analysis, specific respiratory chain defects, and efficacious antioxidant therapy in superoxide dismutase 2 null mice. J Neurochem 88: 657-667.

Hiroi S, Harada H, Nishi H, Satoh M, Nagai R, Kimura A (1999). Polymorphisms in the SOD2 and HLA-DRB1 genes are associated with nonfamilial idiopathic dilated cardiomyopathy in Japanese. Biochem Biophys Res Commun 261: 332-339.

Hommer D, Momenan R, Kaiser E, Rawlings R (2001). Evidence for a gender-related effect of alcoholism on brain volumes. Am J Psychiatry 158: 198-204.

Hommer DW (2003). Male and female sensitivity to alcoholinduced brain damage. Alcohol Res Health 27: 181-185.

Huang MC, Chen CC, Peng FC, Tang SH, Chen CH (2008). The correlation between early alcohol withdrawal severity and 
oxidative stress in patients with alcohol dependence. Prog Neuropsychopharmacol Biol Psychiatry 33: 66-69.

Jernigan TL, Butters N, DiTraglia G, Schafer K, Smith T, Irwin M et al (1991). Reduced cerebral grey matter observed in alcoholics using magnetic resonance imaging. Alcohol Clin Exp Res 15: 418-427.

Ke ZJ, Gibson GE (2004). Selective response of various brain cell types during neurodegeneration induced by mild impairment of oxidative metabolism. Neurochem Int 45: 361-369.

Lecomte E, Herbeth B, Pirollet P, Chancerelle Y, Arnaud J, Musse N et al (1994). Effect of alcohol consumption on blood antioxidant nutrients and oxidative stress indicators. Am J Clin Nutr 60: 255-261.

Lenzen S (2008). Oxidative stress: the vulnerable beta-cell. Biochem Soc Trans 36: 343-347.

Mann K, Ackermann K, Croissant B, Mundle G, Nakovics H, Diehl A (2005). Neuroimaging of gender differences in alcohol dependence: are women more vulnerable? Alcohol Clin Exp Res 29: 896-901.

Martin RC, Li Y, Liu Q, Jensen NS, Barker DF, Doll MA et al (2009). Manganese superoxide dismutase V16A single-nucleotide polymorphism in the mitochondrial targeting sequence is associated with reduced enzymatic activity in cryopreserved human hepatocytes. DNA Cell Biol 28: 3-7.

Maschke M, Weber J, Bonnet U, Dimitrova A, Bohrenkamper J, Sturm S et al (2005). Vermal atrophy of alcoholics correlate with serum thiamine levels but not with dentate iron concentrations as estimated by MRI. J Neurol 252: 704-711.

Meyerhoff DJ, Blumenfeld R, Truran D, Lindgren J, Flenniken D, Cardenas V et al (2004). Effects of heavy drinking, binge drinking, and family history of alcoholism on regional brain metabolites. Alcohol Clin Exp Res 28: 650-661.

Momenan R, Hommer D, Rawlings R, Ruttimann U, Kerich M, Rio D (1997). Intensity-adaptive segmentation T1-weighted magnetic resonance images. Hum Brain Mapp 5: 194-205.

Moreira PI, Santos MS, Oliveira CR, Shenk JC, Nunomura A, Smith MA et al (2008). Alzheimer disease and the role of free radicals in the pathogenesis of the disease. CNS Neurol Disord Drug Targets 7: 3-10.

Paul A, Belton A, Nag S, Martin I, Grotewiel MS, Duttaroy A (2007). Reduced mitochondrial SOD displays mortality characteristics reminiscent of natural aging. Mech Ageing Dev 128: 706-716.

Peng FC, Tang SH, Huang MC, Chen CC, Kuo TL, Yin SJ (2005). Oxidative status in patients with alcohol dependence: a clinical study in Taiwan. J Toxicol Environ Health A 68: 1497-1509.

Pfefferbaum A, Lim KO, Zipursky RB, Mathalon DH, Rosenbloom MJ, Lane B et al (1992). Brain gray and white matter volume loss accelerates with aging in chronic alcoholics: a quantitative MRI study. Alcohol Clin Exp Res 16: 1078-1089.

Pfefferbaum A, Sullivan EV, Mathalon DH, Shear PK, Rosenbloom MJ, Lim KO (1995). Longitudinal changes in magnetic resonance imaging brain volumes in abstinent and relapsed alcoholics. Alcohol Clin Exp Res 19: 1177-1191.

Pfefferbaum A, Sullivan EV, Rosenbloom MJ, Mathalon DH, Lim KO (1998). A controlled study of cortical gray matter and ventricular changes in alcoholic men over a 5-year interval. Arch Gen Psychiatry 55: 905-912.
R Development Core Team (2008). R: A language and environment for statistical computing. R Foundation for Statistical Computing, Vienna, Austria. ISBN 3-900051-07-0, URL http:// www.R-project.org.

Sanchez-Moreno C, Paniagua M, Madrid A, Martin A (2003). Protective effect of vitamin $\mathrm{C}$ against the ethanol mediated toxic effects on human brain glial cells. J Nutr Biochem 14: 606-613.

Schottenbauer MA, Momenan R, Kerick M, Hommer DW (2007). Relationships among aging, IQ, and intracranial volume in alcoholics and control subjects. Neuropsychology 21: 337-345.

Shimoda-Matsubayashi S, Hattori T, Matsumine H, Shinohara A, Yoritaka A, Mori H et al (1997). Mn SOD activity and protein in a patient with chromosome 6-linked autosomal recessive parkinsonism in comparison with Parkinson's disease and control. Neurology 49: 1257-1262.

Shimoda-Matsubayashi S, Matsumine H, Kobayashi T, NakagawaHattori Y, Shimizu Y, Mizuno Y (1996). Structural dimorphism in the mitochondrial targeting sequence in the human manganese superoxide dismutase gene. A predictive evidence for conformational change to influence mitochondrial transport and a study of allelic association in Parkinson's disease. Biochem Biophys Res Commun 226: 561-565.

Stephens M, Donnelly P (2003). A comparison of bayesian methods for haplotype reconstruction from population genotype data. Am J Hum Genet 73: 1162-1169.

Stephens M, Smith NJ, Donnelly P (2001). A new statistical method for haplotype reconstruction from population data. Am J Hum Genet 68: 978-989.

Storz P, Doppler H, Toker A (2005). Protein kinase D mediates mitochondrion-to-nucleus signaling and detoxification from mitochondrial reactive oxygen species. Mol Cell Biol 25: 8520-8530.

Sullivan EV, Rosenbloom MJ, Lim KO, Pfefferbaum A (2000). Longitudinal changes in cognition, gait, and balance in abstinent and relapsed alcoholic men: relationships to changes in brain structure. Neuropsychology 14: 178-188.

Sun AY, Sun GY (2001). Ethanol and oxidative mechanisms in the brain. J Biomed Sci 8: 37-43.

Toyokuni S (2008). Molecular mechanisms of oxidative stressinduced carcinogenesis: from epidemiology to oxygenomics. IUBMB Life 60: 441-447.

Tsai GE, Ragan P, Chang R, Chen S, Linnoila VM, Coyle JT (1998). Increased glutamatergic neurotransmission and oxidative stress after alcohol withdrawal. Am J Psychiatry 155: 726-732.

Uekermann J, Daum I (2008). Social cognition in alcoholism: a link to prefrontal cortex dysfunction? Addiction 103: 726-735.

Weisiger RA, Fridovich I (1973). Mitochondrial superoxide simutase. Site of synthesis and intramitochondrial localization. J Biol Chem 248: 4793-4796.

Yao JK, Reddy RD, van Kammen DP (2001). Oxidative damage and schizophrenia: an overview of the evidence and its therapeutic implications. CNS Drugs 15: 287-310.

Zhang P, Anglade P, Hirsch EC, Javoy-Agid F, Agid Y (1994). Distribution of manganese-dependent superoxide dismutase in the human brain. Neuroscience 61: 317-330.

Supplementary Information accompanies the paper on the Neuropsychopharmacology website (http://www.nature.com/npp) 